\title{
Informal caregivers for stroke survivors: what does occupational therapy offer?
}

\author{
Lyndall Serfontein, B OT (UFS), M OT (UFS). https://orcid.org/0000-000 I-6524-I I 7X \\ Occupational Therapist, Life Pasteur Rehabilitation Hospital, Bloemfontein, Free State
}

*Mia Elsabie Van Schalkwyk, B OT (UFS), M OT (UFS). https://orcid.org/0000-0003-3966-43 I 7
Affiliated Lecturer, Department of Occupational Therapy, Faculty of Health Sciences, University of the Free State.

Marieta Visser, B OT (UFS, MSc OT (Wits). https://orcid.org/0000-0002-8825-4683

Lecturer, Department of Occupational Therapy, Faculty of Health Sciences, University of the Free State

Key message: Viewing informal caregivers from an occupational perspective will equip occupational therapists to offer novel resources and support to the caregivers.

Informal caregivers play an important role in the continuum of care for survivors of stroke and the caregivers' own well-being may therefore have a significant impact on the survivor of stroke. Yet, caregiver training and support often do not fulfil the needs of caregivers and the consequences are often described in literature as 'burden of care'. In this article, we encourage occupational therapists to consider caregiving from an occupational perspective and we propose strategies for the enabling informal caregivers to maintain optimal wellbeing and health by means of increased opportunity for balanced occupational participation.

Key words: stroke, informal caregivers, burden of care, occupational perspective, occupational therapy

\section{INTRODUCTION}

Many people suffer stroke - at least 13.7 million worldwide per year'. For almost as many people, caregiving becomes an occupation, an unexpected responsibility with different tasks often necessitating changes in roles and routines. Occupational therapists are usually involved in the training of caregivers before the survivors of stroke return home from rehabilitation units. However, the content of caregiver training and the resources for ongoing support vary, and caregivers often feel ill-equipped for their task.

Occupational therapists working in rehabilitation are often faced with the following questions: To what extent should caregivers be equipped by the multi-professional team for their role as caregiver? Upon discharge of the patient into their care, what kind of support do caregivers require? What contribution could an occupational therapist make, when considering the caregiver as client?

\section{Informal caregivers - an integral part of the rehabilitation of stroke survivors}

The continuum of care for stroke survivors is often challenged, especially in the context of developing economies. A shortage of stroke-specific policies and protocols, lack of specialised stroke care and rehabilitation, and limited outpatient services and homebased care services can contribute to survivors of stroke not receiving optimal care ${ }^{2}$. Many communities offer little support towards community integration and participation in productive activities such as work or education ${ }^{2,3}$. Consequently, many stroke survivors do not become as healthy, functional and independent as possible and their ongoing need for care and rehabilitation become the responsibility of family and friends, stepping in as informal caregivers ${ }^{2}$.

An informal caregiver mostly refers to an extended family member or friend who volunteers to take care of a stroke survivor after discharge. The caregiver, who is often not trained as a caregiver and not paid for caregiving, takes care of the patient at home. This caregiving usually requires assisting the survivor of stroke in activities not generally expected from a family member or friend ${ }^{4}$.

Forced to take up unplanned roles as informal caregivers, caring for stroke survivors becomes a full time 'job' with many challenges ${ }^{5}$. Stepping into the role of 'hidden healthcare team members', caregivers play a key role in stroke survivors' progress or lack thereof after discharge, regardless of what health systems provide in terms of continuation of therapy ${ }^{7}$. Consequently, if stroke survivors do not reach optimal functional outcomes, they need more input from caregivers, who eventually experience undue strain and a range of associated issues.

\section{Caregiving as an occupation - positioning the caregiver within the domain of occupational therapy}

Caregiving as a role necessitates specific occupations. Within the domain of occupational therapy, as outlined by the $3^{\text {rd }}$ edition of the occupational therapy practice framework ${ }^{8}$, published by the American Occupational Therapy Association in 2014 , the occupational therapist needs to consider the caregiver's ( $I$ ) occupations, (2) client factors, (3) performance skills, (4) performance patterns; and (5) contexts and environments.

\section{Occupations}

"You do things you would rather not do and almost never get to do the things you want to do. It is as though your life has been completely taken over by another." "4:4 (emphasis added). Caregiving, as a co-occupation ${ }^{10}$ is all about 'doing' - doing things 'for, to, with and because of' another person". Both parties are affected by the other's performance of the activity ${ }^{10}$. 
Many caregivers experience occupational loss soon after taking up the role of caregiver ${ }^{3}$ - limiting or quitting participation in activities previously found meaningful and important, including having to give up paid employment ${ }^{7}$, social and recreational activities, and daily routines. Consequences of occupational loss include higher stress levels, lower energy levels, and decreased psychological well-being ${ }^{3}$. Since caregivers often feel overwhelmed and socially isolated ${ }^{12}$, their decreased social interaction results in a further and gradual decay of social support systems.

Occupational therapists need to understand that the caregiverpatient situation may pose both potential and risk for both parties. Both the caregiver and stroke survivor are occupational beings, with the need and right to participate in activities that they find meaningful. Both are at risk of experiencing occupational injustice and imbalance, since their opportunities to participate in meaningful activities are, to a great extent, influenced by another. Occupational therapy has the potential to assist caregivers in resuming participation in 'valued activities'12, to maintain or regain a sense of occupational balance and should prioritise this issue - also in the best interest of the stroke survivor, who will benefit from a healthy caregiver.

\section{Client factors}

Considering the reciprocal effect between occupation and health, we need to consider how client factors (including current health status and predisposition to certain conditions ) relate directly to the caregiver's experience of and performance of caregiving, but also how the occupation of caregiving may have an impact on the health of a caregiver. Emotional challenges are often caused by factors such as their own health-related problems ${ }^{13}$, resulting in physical, psychological, emotional, social, as well as financial challenges ${ }^{14}$. These in turn, may lead to tiredness, anxiety, despair, frustration, and isolation as a result of caregiving-related duties ${ }^{7}$.

Perhaps in relation to their own values and beliefs, caregivers frequently perceive that their new responsibilities and the patient's needs should enjoy preference above their own, causing them to neglect their own occupational needs ${ }^{3,12}$.

When planning a patient's discharge, therapists are usually attentive in regard to what skills and abilities potential caregivers would require to be successful in their tasks. We urge therapists to also consider client factors which may complicate the caregiver's experience and performance of caregiving. For example, caregivers with a history of lower back pain, may require more guidance in terms of ergonomic principles to follow during tasks involving any transfers. Similarly, caregivers with a history of depression or anxiety will need specific guidance on self-care strategies and sources of support to enable them to adjust successfully to the role of caregiver.

\section{Performance skills}

Caregivers of stroke survivors frequently report that they do not feel equipped to handle stroke survivors' special bodily, psychological, and mental requirements after discharge ${ }^{13}$. We believe that carefully tailored caregiver training may at least partially fill this gap in terms of improving skills, to ensure longevity of caregiving and optimal functional outcomes for the stroke survivor.

Motor skills refer to how persons "interact with and moves task objects and self around the task environment" ${ }^{8: 525}$, for example how they position, align and coordinate body parts to manipulate the objects involved in tasks. In preparation for the safe execution of the tasks involved in caregiving, caregivers may need training in specific motor skills. Process skills relate to how the person "organises actions in a timely and safe manner" ${ }^{8: 525}$, by means of skills such as choosing, initiating, organising and gathering. For caregivers, training in sequencing and organisation of objects and tasks may make a considerable difference in the ease, efficacy and safety with which they perform tasks. Finally, social interaction skills are those skills observed in social exchanges and include a variety of skills from the 'basic' such as speaking fluently and making eye contact, to more 'complex' skills such as placing self, timing response and using matching language. If a caregiver is expected to work with a patient with challenges such as hemi-agnosia or aphasia, training in where to place oneself or how to time a response might make a world of difference to their own wellbeing and that of the patient.

\section{Performance patterns}

The new routines inherent to caregiving may leave little opportunity for a caregiver's own choice of activities, their own valuable and useful habits, routines and rituals ${ }^{12}$. Notwithstanding the impact of a new structure to every day, the caregiver may also have to concede the stroke survivor's preferences in terms of habits, routines and rituals. As mentioned before, caregivers frequently perceive that stroke survivors' needs should supersede their own, causing them to neglect their occupational needs. Careful guidance from an occupational therapist could assist the caregiver to tailor their performance patterns, satisfying both their own needs and those of the stroke survivor, ultimately affording both the opportunity for occupational balance. An example here might be to encourage the care giver with lower back pain, to build a new routine of doing appropriate stretches after specific tasks, or when the patient is resting. This endeavour by the therapist would, however, only be effective if the caregiver truly understands the importance of selfcare and the long-term effect on both themselves and the patient.

\section{Contexts and environments}

The role of the caregiver, as mentioned above, implies that one is forced to share one's context, environment, one's life, with the stroke survivor ${ }^{13,14}$. The caregiver's own context, apart from the survivor of the stroke, also impacts directly on their experience of strain. If the caregiver has little support, or their duties are not shared with other caregivers, they may experience a higher burden of care, related to how their feelings of isolation increase ${ }^{14}$.

Survivors of stroke and their caregivers often experience a significant disparity between the simulated environment in rehabilitation units and their home environment ${ }^{15}$. Although therapists may have very little control about the contexts and environments the patients and caregivers return to, they should focus on presenting training and rehabilitation in ways that are relatable and transferable to the home environment, in order to reach optimal functional outcomes.

Caregivers are often reported as experiencing many challenges - emotional, financial, and even health-related, whilst feeling poorly supported $7,13,14$. Literature on 'burden of care' makes an important contribution to occupational therapy's understanding of caregiving as an occupation. However, if we plan to truly impact a caregiver's wellbeing, we need to approach the person's whole life situation (including perceived burden of care), from an occupational point of view.

\section{RECOMMENDATIONS}

Literature provides assorted recommendations for caregiver education and support. Yet, working in health systems that often limit the kind of support professionals can offer to informal caregivers, occupational therapists need to engage novel strategies. Our recommendations are based on two principles. Firstly, the caregiver is approached as an occupational being, requiring recommendations to be made from an occupational point of view; and secondly, the recommendations are made in consideration of contexts where organised support are not readily accessible.

Viewing caregivers as both an important part of the therapy team (to the benefit of the patient), as well as a client (assessing and supporting them in their specific occupational needs), we encourage occupational therapists to consider:

a) Providing continuity of care necessary for both the caregiver and patient following the stroke survivor's discharge from formal care. To facilitate this kind of support and resources, occupational therapists need to consider novel strategies of being available remotely for consultation, coaching and training (i.e. telephone consultations, video calls, and web-based modules). Remote access to consultation and coaching may 
have the added benefit of offering support in the caregiver and patient's real environment, rather than the simulated environment of the rehabilitation unit.

b) Ensuring caregivers understand the importance of remaining engaged in activities and occupations they find meaningful ${ }^{12}$. Consultation with an occupational therapist, even remotely as recommended above, may assist the caregiver to consider 'reprioritising' of previous and current activities, and embracing old activities in new ways.

c) Advocating for regular respite for caregivers ${ }^{9,12}$ to allow them the opportunity to engage in activities not revolving around the patient. Again, even telephonic consultation may be helpful in assisting the caregiver to make decisions regarding respite care, and then assisting in the planning of respite care for the patient.

d) Lobbying on a policy level for reimbursement of consultation and coaching services which is focused on the caregiver. As reimbursement of therapy services are usually limited to direct intervention to patients, research aimed at describing the role of occupational therapy in caregiver training and the effect on functional outcomes of the patient is needed.

e) Investigating how caregivers' 'roles reinforce their values and beliefs' ${ }^{8}$, and vice versa. If caregivers understand how their own values and beliefs impact their occupational participation, and how being a caregiver may conflict with or support their values and beliefs, they may be able to better understand the importance of finding ways to continue participation in activities in which they find meaning.

\section{SUMMARY}

This opinion piece suggests that caregivers play an integral part of the rehabilitation of survivors of stroke and often become the extension of an incomplete and insufficient rehabilitation service. Optimal management of stroke survivors needs to include serious consideration of the occupational disruption and challenges anticipated for their informal caregivers, to plan appropriate caregiver preparation and education, and ensure ongoing support.

\section{REFERENCES}

I. Feigin VL, Nichol E, Alam T, Bannick MS, Beghi E, Blake N, Culpepper WJ, Dorsey ER, Elbaz A, Ellenbogen RG and Fisher JL. Global, regional, and national burden of neurological disorders, 1990-2016: a systematic analysis for the Global Burden of Disease Study 2016. Lancet Neurol. 2019; 18(5): 459-480.

https://doi.org/10.1016/S1474-4422(18)30499-X

2. Pandian JD, Gandhi DB, Lindley RI and Bettger JP. Informal Caregiving: A Growing Need for Inclusion in Stroke Rehabilitation. Stroke. 2016; 47(I2): 3057-3062.

http://doi.org/I0.1 I6I/STROKEAHA. I I6.01370 I

3. Kniepmann K. Female family carers for survivors of stroke: occupational loss and quality of life. Br J Occup Ther. 20 I 2; 75(5): 208-2 16. http://doi.org/10.4276/0308022 I 2X1336/458480207

4. Dibsdall L and Rugg S. Carers' perspectives on their needs and local authority occupational therapy practice. $\mathrm{Br} J$ Occup Ther. 2008; 7I(7): 277-285.

http://doi.org/I0.1 I 77/030802260807/00704

5. Angelo J and Wilson L. Exploring occupation roles of hospice family caregivers from Mãori, Chinese and Tongan ethnic backgrounds living in New Zealand. Occup Ther Int. 20I4; 2 I (2): 8I-90. http://doi.org/10.1002/oti. I 367

6. Moghimi C. Issues in caregiving: The role of occupational therapy in caregiver training. Top Geriatr Rehabil. 2007; 23(3): 269-279. http://doi.org/10.1097/0I.TGR.0000284770.39958.79

7. Thomas $M$ and Greenop K. Caregiver experiences and perceptions of stroke. Health SA Gesondheid (Online). 2008; I3(I): 29-40. http://doi.org/l0.4102/hsag.vl 3il.255

8. American Occupational Therapy Association. Occupational therapy practice framework: domain and process (3rd ed.). Am J Occup Ther. 2014; 68(Suppl I): SI - S48.

http://doi.org/10.5014/ajot.2014.682006
9. Owusu-Ansah FE. Sharing in the life of the person with disability: A Ghanaian perspective. Afr J Disabil. 2015; 4(I). http://doi.org/10.4I02/ajod.v4il.I85

10. Dalvand H, Hosseini SA, Rassafiani M, Samadi SA, Khankeh HR and Kelly G. Co-occupations: The caregiving challenges of mothers of children with cerebral palsy. Br J Occup Ther. 20I5; 78(7): 450-459. http://doi.org/I0.I I 77/03080226I4562793

II. Doidge K. Co-occupation categories tested in the mothering context (Masters dissertation). 2012. http://hdl.handle.net/II604/47

12. Jellema S, Wijnen, MA, Steultjens EM, Nijhuis-van der Sanden MW and van der Sande R. Valued activities and informal caregiving in stroke: a scoping review. Disabil Rehabil. 201 9; 4I (I8): 2223-2234. http://doi.org/I0.1080/09638288.2018.1460625

13. Shyu YIL, Chen MC, Chen ST, Wang HP and Shao JH. A family caregiver-oriented discharge planning program for older stroke patients and their family caregivers. Journal of Clinical Nursing. 2008; I7(18): 2497-2508. http://doi.org/10.1 I I I/j. I 365-2702.2008.02450.x

14. Sedrez-Celich KL, Maschio G, da-Silva-Filho CC, Gaffuri-da-Silva T and Silva-de-Souz S. Influence of family support in burden of caregivers of individuals with cerebrovascular accident sequelae. Invest Educ Enferm. 2016; 34(3): 544-550.

http://doi.org/10.17533/udea.iee.v34n3al4

15. Ekstam L, Johansson U, Guidetti S, Eriksson G and Ytterberg C. The combined perceptions of people with stroke and their carers regarding rehabilitation needs I year after stroke: a mixed methods study. BMJ open. 20I5; 5(2): e006784. http://doi.org/10.1 I36/bmjopen-2014-006784

\section{AUTHOR CONTRIBUTIONS}

This article was developed from an original article submitted by Lyndall Serfontein as part of her dissertation (Masters in Occupational Therapy at the University of the Free State). Mia van Schalkwyk and Marieta Visser assisted in the rewriting of the article, and all three authors agreed to this version of the article.

Corresponding Author

* Mia van Schalkwyk

Email: miavs8I@icloud.com 